\title{
Robust Model for Signature Recognition Based on Biological Inspired Features
}

\author{
Reza Ebrahimpour, Ali Amiri, Masoom Nazari and Alireza Hajiany
}

\begin{abstract}
This paper introduces a new and robust model for signature recognition by means of features inspired by the human's visual ventral stream. A feature set is extracted by means of a feed-forward model which contains illumination and view invariant $\mathrm{C2}$ features from all images in the dataset. Also we use from Linear Discrimniant Analysis (LDA) to reduce the dimension of $\mathrm{C} 2$ feature vectors that is derived from a cortex-like mechanism. Then we utilized standard K-Nearest Neighbor (KNN) as classifier. The effectiveness of the approach is evaluated on an experimental signature database. By this new effort the rate of signature recognition is significantly high toward other models.
\end{abstract}

Index Terms - Signature Recognition, Visual Ventral Stream, HMAX, C2 features.

\section{INTRODUCTION}

Handwritten signature is one of the biometrics of human's behavior which is acceptable as a symbol for authorization and identification. Many documents such as forms and bank cheques necessitate the signing of a signature. Therefore it is essential to recognize the signatures, with high accuracy and no time consuming processes.

Handwritten signature recognition can be divided into on-line (or dynamic) and off-line (or static) recognition. On-line recognition refers to a process that the signer uses a special pen called a stylus to create his or her signature, producing the pen locations, speeds and pressures, while off-line recognition just deals with signature images acquired by a scanner or a digital camera [1].

Off-line signature recognition is an important problem. Unlike the on-line signature, where dynamic aspects of the signing action are captured directly as the handwriting trajectory. Handwriting features, such as the handwriting order, writing-speed variation, and skillfulness, need to be recovered from the grey-level pixels [1]. During the last few years, researchers have made great efforts on off-line signature recognition. Buryne tried to apply elastic image matching in signature recognition (Buryne and Forre, 1986) [17].Ng and $\mathrm{He}$ used a neural network expert system to identify Chinese signature (Ng and Ong, 1993 ; He et al, features to compute the Euclidian distance of two signatures

Reza. Ebrahim pour. Assistant Professor, Department of Electrical Engineering, Shahid Rajaee University, Tehran, P. O. Box 16785-136, Fax : +982122970006 Iran; research fields: human and machine vision, neural networks and pattern recognition.

Ali. Amiri. Master student, research fields: image processing, pattern recognition and neural networks. (E-mail: evinar@gmail.com).

Masoom. Nazari. Master student, research fields: image processing, pattern recognition and neural networks. (E-mail: innocent@gmail.com).

Alireza Hajiany, BS student, research fields: signal processing, ensemble neural networks, face recognition.
(Yingyong and Hunt, 1994) [18]. 2002) [19]. Yingyong and Hunt used some global and grid Models based on texture analyses were also applied in the area, with works by Liu et al. (1997) [20] and Liu (1997) [21]. Peter Shaohua discussed the possibility of applying wavelet on signature verification in their paper of 1999 (Peter Shaohua et al, 1999) [22]. Justino and El-Yacoubi developed a signature recognition system based on Hidden Markov Model (HMM) (Justino et al, 2001 [23], and El-Yacoubi et al, 2000) [24]. Wan and Lin presented an off-line signature recognition system that only requires the genuine signatures of a new user (Wan et al, 2003) [25]. Hairong Lv and Wenyuan Wang, used from both static features and dynamic features and support vector machine as classifier for verify the Chinese signatures (Hairong Lv and Wenyuan Wang, 2005) [1]. M. TaylanDas, L. CananDulger, proposed a new model based on a Neural Network (NN) for off-line signature verification [12].

Riesenhuber and Poggio proposed a model that is motivated from a quantitative theory of ventral stream of visual cortex [2]. This system has high performance at complex scenes. In these scenes object recognition must be robust respect to pose, scale, position, rotation and Image condition (lighting, camera characteristics and resolution). The simplest version of the model consists of four layers that try to summarize a core of well-accepted facts about the ventral stream in the visual cortex [2], [4] and [5].

In this paper, a robust model for off-line signature recognition is proposed, based on KNN. After extracting C2 features and reduction the dimension by LDA, the KNN classifier is employed to identify the signature images.

The remainder of the paper is organized as follows: Section II introduces the database used in this experiment; Section III discusses about our proposed model; In Section IV our technique for feature extraction is presented; In Section V strategies and experimental results of signature recognition by this model are presented; And Section VI presents the conclusion and future work.

\section{DATA BASE}

The signature database used in this experiment is an experimental database, which is collected by students of Shahid Rajaee Teacher Training University (SRTTU) of Tehran, Iran, in august 2007. It consists of 282 signature images, scanned at a resolution of $300 \mathrm{dpi}, 8$-bit grey-scale. They are organized into 47 sets. There are 6 signatures in each set. Each volunteer was asked to sign his or her own signatures 6 times on a white paper as shown in Fig. 1. 


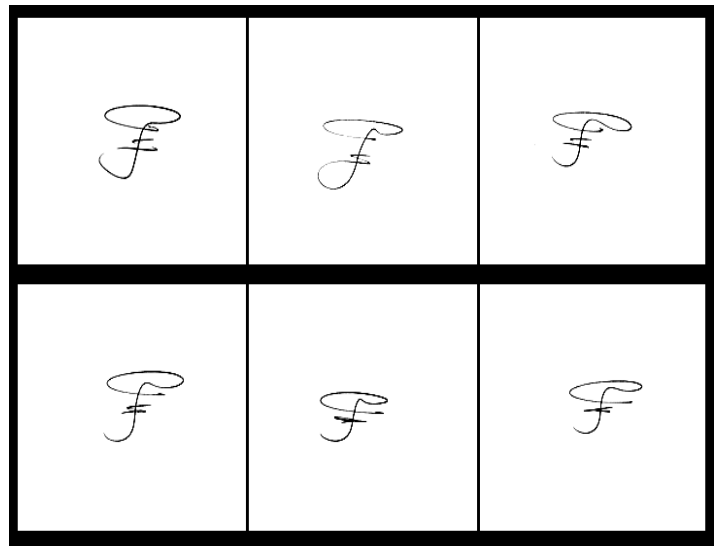

Fig. 1. Some samples of Signature Database used to train and test our proposed model.

\section{OUR PROPOSED MODEL}

In this paper the new model for signature recognition based on biologically motivated feature extraction is proposed. First the feature vectors for train and test are extracted for each signature image by using HMAX model (this model is proposed at 1996 by Poggio and Serre in Masachossete Inistitute of Technology (MIT) (See fig. 2)).

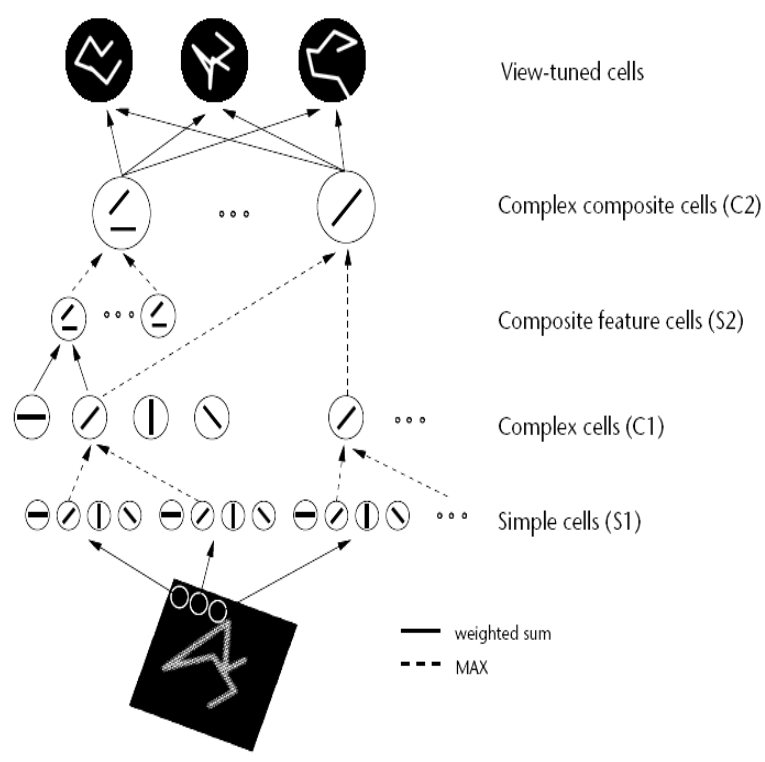

Fig. 2. The schematic of HMAX model [5].

The HMAX feature extraction system follows the feedforward mechanism of object recognition in cortex. The process of visual system in human and privates is hierarchical, Aiming to build invariance to position and scale first and then to viewpoint and other transformations. Along the hierarchy, response of receptive fields of the neurons is measured and used for constructing this model.

This model consists of four layers of computational units where simple $\mathrm{S}$ cells alternate complex $\mathrm{C}$ cells. The inputs of the $\mathrm{S}$ units are combined with a bell-shaped function to increase selectivity. The $\mathrm{C}$ units pool their inputs through a maximum (MAX) operation, and it cause to increasing of invariance. These layers are derived from the experiments in monkey IT cortex (See fig. 2).
Then the dimension of feature vectors is reduced because of reduction time of computation and saving time of recognition. At the end the dimensionally reduced feature vectors passed to the classifier. Biologically motivated feature extraction, describes a feature extracting system that derives from a feedforward model of visual cortex, illumination and view invariant $\mathrm{C} 1$ features from all images in the dataset [2].

\section{FEATURE EXTRACTION}

Feature extraction means extracting of powerful features in different cases of recognition and recognition, Such as signature recognition. And the way of feature extraction is very important task.

In this paper we use from biologically motivated feature extraction, which is inspired by ventral stream of Human's Visual System (HVS).

After extracting feature vectors, the dimension of this vectors reduced by using of Principle Component Analysis (PCA) technique [3]. PCA extracts eigenvectors from covariance matrix to find the principle component of the distribution of signatures. Each individual signature in the dataset can then be approximated by a linear combination of the largest eigenvectors.

Recent researches on ventral stream of visual cortex in human and primates led to different theories about dividing the visual cortex into different levels that information encode in that levels. Visual processing in ventral stream of cortex is hierarchical and feedforward process.

At the first step, the receptive fields of the Simple neurons (S units) combine their inputs with a bell shaped tuning function to increase the selectivity, and the Complex neurons (C units) pull their inputs with maximum operation, and this is result in increasing of invariance. Neurons in visual V4 area exhibit partially complex shapes with information about the structural description of the represented feature [14].

Learning to recognize 3D object doing in the final stage of processing (e.g. inferotemporal cortex (IT) area).

Riesenhuber and Poggio proposed a model that is motivated from a quantitative theory of ventral stream of visual cortex [4]. This system has high performance at complex scenes. In these scenes object recognition must be robust respect to pose, scale, position, rotation and Image condition (Lighting, camera characteristics and resolution). The simplest version of the model consists of four layers that try to summarize a core of well-accepted facts about the ventral stream in the visual cortex.

At the first, simple $\mathrm{S}$ units combine their inputs with a bell-shaped function to increase selectivity, and complex $\mathrm{C}$ units pool their inputs through a maximum operation, therefore increasing invariance. Simple neurons divide in two part, S1 units and S2 units.

\section{A. S1 Units}

$S 1$ units take the form of Gabor functions, which have been shown to provide a good model of cortical simple cell receptive fields [3]. And are described by the following equation: 


$$
\begin{aligned}
& F(x, y)=\exp \left(-\frac{\left(x_{0}{ }^{2}+\gamma^{2} y_{0}{ }^{2}\right)}{2 \sigma^{2}}\right) \times \cos \left(\frac{2 \pi}{\lambda} x_{0}\right), \\
& x_{0}=x \cos \theta+y \sin \theta \text { And } y_{0}=-x \sin \theta+y \cos \theta,(2)
\end{aligned}
$$

All filter parameters, i.e., the aspect ratio, $\gamma=0.3$, the orientation $\theta$, the effective width $\sigma$, the wavelength $\lambda$ as well as the filter sizes $s$ were adjusted so that the tuning properties of the corresponding S1 units match the bulk of V1 parafoveal simple cells based on data from two groups
[9]-[11]. Setting of parameters is done by using of biological experiments on monkeys to obtain the tuning properties.

In this experiment $\mathrm{S} 1$ units are 3? to 15? 5 pixels (See table. I) in steps of 2 pixels. To keep the number of units tractable we considered 4 orientations ( 0 ? 45? 90)瘾nd 135 ? thus leading to 28 different $S 1$ receptive field types total (7 scales $\times 4$ orientations).

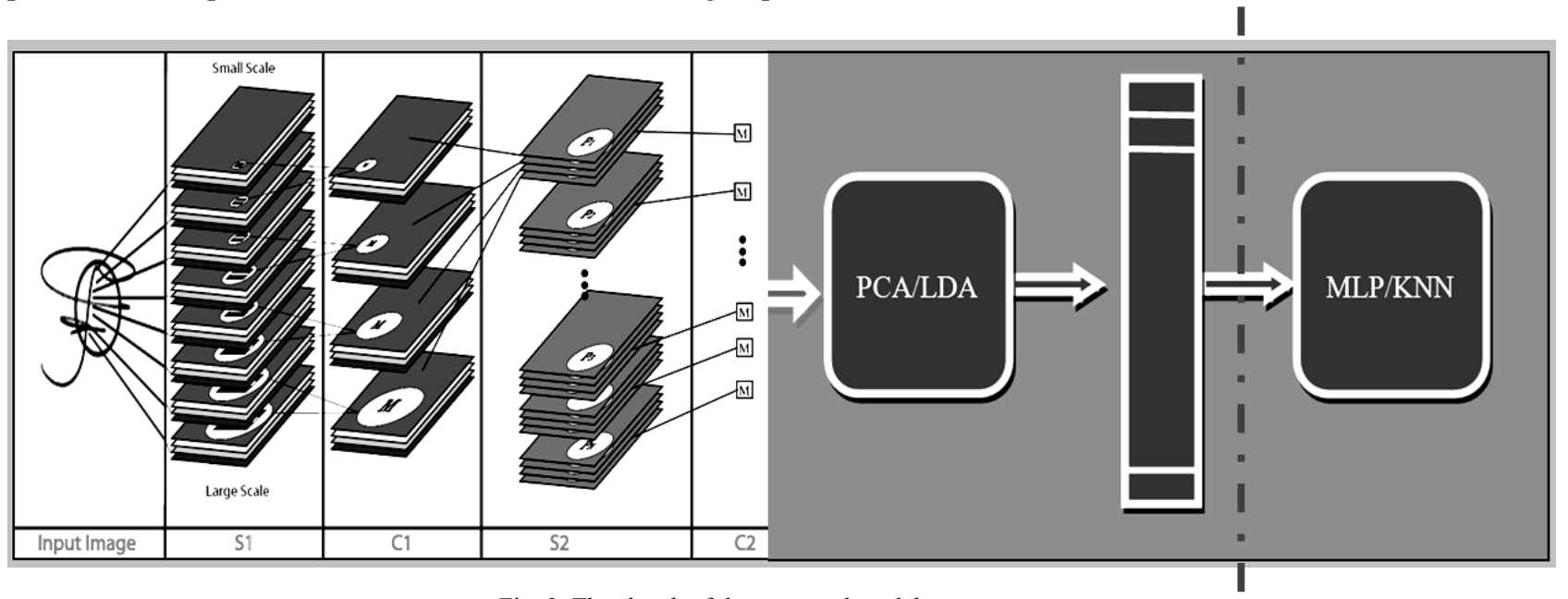

Fig. 3. The sketch of the proposed model.

\section{B. C1 Units}

These units Are more complicated and have larger receptive fields than $\mathrm{S} 1$ units have response to bars or edges anywhere into their receptive field. And C1 units pool the outputs of S1 units by using a MAX operation. That is, the response $r$ of a complex unit corresponds to the response of the strongest of its $m$ afferents $\left(x_{1}, x_{2}, \ldots, x_{m}\right)$ from the previous $S 1$ layer such that:

$$
r=\max _{j=1 \ldots m} x_{j}
$$

\section{S2 Units}

In this layer, units pool the outputs of the $\mathrm{C} 1$ layer from a spatial neighborhood for each orientations, the behavior of S2 units is as Radial Basis Function (RBF) units [2].

The response of each S2 unit depends in Euclidean distance between a new input and prototypes that before stored such that:

$$
r=\exp \left(-\beta\left\|X-P_{i}\right\|^{2}\right),
$$

Where $\beta$ is the sharpness of tuning and $P_{i}$ is the sorted prototype and $\mathrm{X}$ is the new input image.

\section{C2 Units}

At the end of feature extraction by HMAX, the $\mathrm{C} 2$ units are extracted as shift and scale-invariant units by taking a global maximum (See Eq.1) over all scales and positions for S2 units. The S2 measures the rate of match between a stored prototype and an input image for finding the best match and discard the rest.

The result is $\mathrm{N} \mathrm{C} 2$ feature vector; That $\mathrm{N}$ corresponds to the number of prototypes extracted during learning stage.

\section{E. The learning stage}

In this stage $\mathrm{N}$ prototype are selected by a simple sampling, that the prototypes are selected randomly at various sizes and positions from a target set of positive images. The prototypes are extracted in the level of $\mathrm{C} 1$ layer for 4 orientations (e.g. $\mathrm{n} \times \mathrm{n} \times 4$ elements). In this experiment patches of 4 different sizes are extracted $(n=3,5,7,9,11,13,15)$.

\section{F. Classification stage}

Images are propagated through the architecture described in Fig. 1. The C1 and C2 Standard Model Features (SMFs) are then extracted and further passed for reduction the dimension by Linear Discrimination Analysis (LDA) or Principle Component Analysis (PCA). At the end the NC2 output feature vectors are passed to a classifier such as K-Nearest Neighbor (KNN) or Multi Layer Perceptron (MLP) [16].

In over all our proposed model, for $\mathrm{KNN}$ classifier $\mathrm{K}=1$ and initial weights for MLP are randomly selected.

Furthermore some of other algorithms of feature extraction such as LDA, PCA and HIST [8] are tested for compare with biologically motivated feature extraction. And at the same condition of initial weights for MLP classifier and the same K for $\mathrm{KNN}$, the results are compared in table. I.

\section{EXPERIMENTAL RESULTS}

In our experiments, the main task is recognition of signatures of different people from each other. We use from an experimental database which is described in section. 2. In This database signatures are organized into 47 sets. Each set contains 6 signatures from one volunteer. And in this experiment half of each set is used for training and the rest is used for testing. (See fig. 1). Our model based on extracting 
features from signature images by using of Standard Model Features (SMFs) and Linear Discrimination Analysis (LDA) as feature extractor. Best rate of recognition, which after ten runs is $\mathbf{9 7 . 6} \%$, if we use from K-Nearest Neighbor $(\mathrm{KNN})$ as classifier. The performance of some of different models for feature extraction and classification are shown in table. I. And results showed that using Standard Model Features (SMFs) and Linear Discrimination Analysis (LDA) as feature extractor, and use from Multi Layer Perceptron (MLP) as classifier, after ten runs, led to the rate of recognition decreases to $\mathbf{9 2 . 2 1 \%}$. And by using Standard Model Features (SMFs) and KNN classifier, the rate of recognition is $91.00 \%$.

This model is good way for obtain the high recognition of signatures toward other common models. Our model is based on Human Visual System (HVS), and the HVS is very accurate system of object recognition, therefore it can recognize the signatures stronger than other models.

\section{CONCLUSION}

We proposed a model based on Human Visual System (HVS) to achieve high performance signature recognition. We compared it with other models of signature recognition to demonstrate the superior performance. And experimental results supported our claim that it is better than other common models for signature recognition. By applying our model the average of signature recognition was increased.

HMAX appears to be powerful tool to approach more robust signature recognition models. In future work, we plan to explore more complicated models of feature extraction by using Standard Model Features (SMFs) and using mixture of other feature extractors for leading to the high performance signature recognition.

TABLE I. ReCognition RATE of DifFerent Signature ReCognition Models. In Each Row, ReCognition Rates Which Is
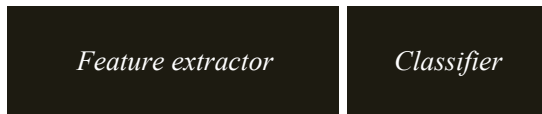

C2 SMFs+ LDA

C2 SMFs
LDA
PCA

KNN

KNN

$\mathrm{KNN}$

HIST

\begin{tabular}{|r|}
\hline LDA \\
\hline PCA \\
HIST \\
\hline C2 SMFs
\end{tabular}

C2 SMFs

C2 SMFs+ LDA

$\mathrm{KNN}$

$\mathrm{KNN}$

MLP

MLP
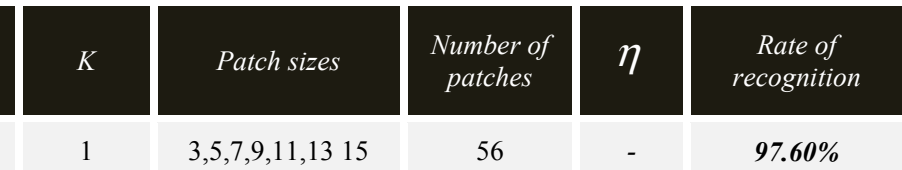

56

$3,5,7,9,11,1315$

\begin{tabular}{|l|l|}
\hline MLP & - \\
\hline MLP & - \\
\hline MLP & - \\
\hline
\end{tabular}

\section{REFERENCES}

[1] Hairong Lv, Wenyuan Wang, Chong Wang, Qing Zhuo. "Off-line Chinese signature verification based on support vector machines," Pattern Recognition Letters 26, 2005, pp. 2390-2399.

[2] Thomas Serre, Lior Wolf, Stanley Bileschi, Maximilian Riesenhuber, and Tomaso Poggio, Member, IEEE, "Robust Object Recognition with Cortex-like Mechanisms, " IEEE transactions on pattern analysis and machine intelligence, august. 2006.

[3] M.A. Turk, A.P. Pentland, "Eigen faces for recognition," J. Cognit. Neuroscience. Vol. 3 (1), 1991, pp. 71-86.

[4] Serre, Kouh, M, Cadieu, C, Knoblich, U, Kreiman, G., Poggio, T, “A Theory of Object Recognition: Computations and Circuits in the Feedforward Path of the Ventral Stream in Primate Visual Cortex," AI Memo 2005-036/CBCL Memo 259. Massachusetts Inst. Of Technology, Cambridge. 2005.

[5] Riesenhuber, M. and Poggio, T. "Hierarchical models of object recognition in cortex," Nature Neurosis. 1999.

[6] R. DeValois, E. Yund, and N. Hepler, "The orientation and direction selectivity of cells in macaque visual cortex," Vis. Res., vol. 22, 1982, pp. 531-544.

[7] P. H. Schiller, B. L. Finlay, and S. F. Volman, "Quantitative studies of Single-cell properties in monkey striate cortex," III. Spatial frequency, J. Neurophysiol, vol. 39, No. 6, 1976, pp. 1334-1351.

[8] Sherif Abdleazeem · Ezzat El-Sherif, "Arabic handwritten digit recognition,” IJDAR, Vol. 11, 2008, pp. 127-141.
[9] R. DeValois, D. Albrecht, and L. Thorell, "Spatial frequency selectivity of cells in macaque visual cortex," Vis. Res, vol. 22, 1982. pp. 545-559.

[10] R. DeValois, E. Yund, and N. Hepler, "The orientation and direction selectivity of cells in macaque visual cortex," Vis. Res., vol. 22, 1982, pp. 531-544.

[11] P. H. Schiller, B. L. Finlay, and S. F. Volman, "Quantitative studies of single-cell properties in monkey striate cortex III. Spatial frequency," J. Neurophysiol, vol. 39, no. 6, 1976, pp. 1334-1351.

[12] M. TaylanDas, L. Canan Dulger, "Signature verification (SV) toolbox: Application of PSO-NN," Engineering Applications of Artificial Intelligence 22, 2009, pp. 688-694.

[13] I.D. Trier, A.K. Jain, "feature extraction methods for character recognition- a survey," Pattern Recognition, vol. 38, no.12, 2005, pp. 2270-2285.

[14] Kai Huang, Hong Yan, "Off-line signature verification using structural feature correspondence," Pattern Recognition, vol. 35, 2002, pp. 2467 $-2477$.

[15] Alessandra Lumini, Loris Nanni, "Ensemble of on-line signature matchers based on Over Complete feature generation," Expert Systems

[16] with Applications 36, 2009, 5291-5296.

[17] Gonzalez, C., Wintz, P, "Digital Image Processing," second Ed. Addison-Wesley, 1987.

[18] Buryne, P.de, Forre, R., 1986. "Signature verification with elastic image matching," In: International Carnahan Conference on Security Technology Gothenburg, Sweden, August 12-14, 1986. 
[19] Yingyong, Q., Hunt, B.R, "Signature verification using global and grid features," Pattern Recognition vol. 22 (12) Great Britain, 1994, pp. $1621-1629$.

[20] Ng, G.S., Ong, H.S, “A neural network approach for offline signature verification," In: Proceedings of Computer, Communication, Control and Power Engineering, TENCON 93. IEEE Region 10 Conference, vol. 2, October 19-21, 1993, pp. $770-773$.

[21] Liu, Chenglin, "Writer identification by multichannel decomposition and matching,"Acta Automatica Sinica, 1997.

[22] Liu, Chenglin, Dai, Ruwei, Liu, Yingjian, "Modified Wigner distribution and application to writer identification," Chinese Journal of Computers 20 (11), 1997.

[23] Peter Shaohua, Deng, Hong-Yuan Mark, Liao, Chin Wen, Ho, Hsiao-Rong, Tyan, "Wavelet-Based Off-Line Handwritten Signature Verification," Computer Vision and Image Understanding 76 (3), 1999 , pp. 173-190.

[24] Justino, E.J.R., Bortolozzi, F, Sabourin, R., 2001. "Off-line signature Verification using HMM for random, simple and skilled forgeries," Document Analysis and Recognition, 2001. In: Proceedings of Sixth International Conference, September 10-13, 2001, pp. 1031-1034

[25] El-Yacoubi, A., Justino, E.J.R., Sabourin, R., Bortolozzi, F, 2000. "Off-line signature verification using HMMs and cross validation," Neural Networks for Signal Processing X 2000. In: Proceedings of the IEEE Signal Processing Society Workshop, vol. 2, December 11-13, 2000, pp. 859-868.

[26] Wan, L., Lin, Z. C., Zhao, R. C., "Off-line signature verification incorporating the prior model. Machine Learning and Cybernetics,' International Conference, vol. 3, November 2-5, 2003, pp. 1602-1606.

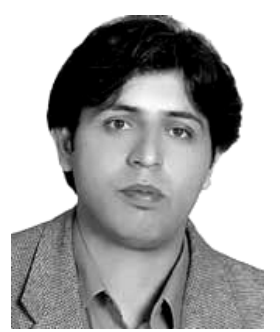

Reza Ebrahimpour was born in Mahallat, Iran, in July 1977. He received the BS degree in electronics engineering from Mazandaran University, Mazandaran, Iran and the MS degree in biomedical engineering from Tarbiat Modarres University, Tehran, Iran, in 1999 and 2001, respectively. He received his $\mathrm{PhD}$ degree in July 2007 from the School of Cognitive Science, Institute for Studies on Theoretical Physics and Mathematics, where he worked on view-independent face recognition with Mixture of Experts. His research interests include human and machine vision, neural networks, and pattern recognition.

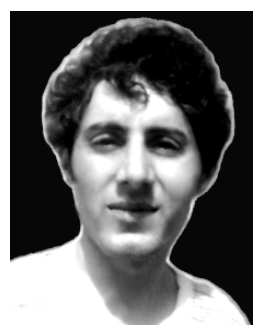

Ali Amiri was born in Ilam, Iran, in August 1984. Master student, Electrical Engineering Department of Shahid Rajaee University Tehran, Iran from 2009 up now. His research interest includes image processing, pattern recognition and neural networks.

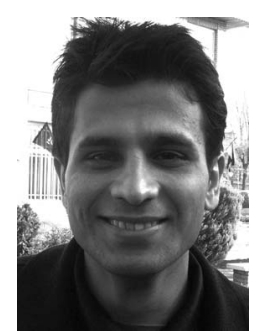

Masoom Nazari was born in Marand, Iran, in September 1984. Master student, Electrical Engineering Department of Shahid Rajaee University Tehran, Iran from 2009 up now. His research interest includes image processing, pattern recognition and neural networks.

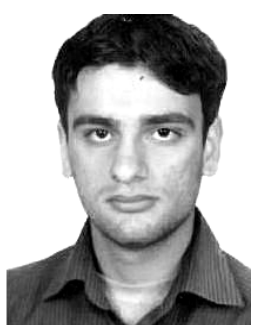

Alireza Hajiany was born in Booshehr, Iran, in August 1987. BS student, Electrical Engineering Department of Shahid Rajaee University Tehran, Iran from 2004 up now. His research interest includes signal processing, ensemble neural networks, face recognition. 